\title{
ACCELERATION ENVIRONMENT OF THE INTERNATIONAL SPACE STATION
}

AIAA-2009-0957

\author{
Kevin McPherson \\ NASA Glenn Research Center, Mail Stop 77-7, 21000 Brookpark Rd., Cleveland, OH 44135-3191, USA \\ Eric Kelly \\ ZIN Technologies, Inc., Mail Stop 77-7, 21000 Brookpark Rd., Cleveland, OH 44135-3191, USA \\ Jennifer Keller \\ ZIN Technologies, Inc., Mail Stop 77-7, 21000 Brookpark Rd., Cleveland, OH 44135-3191, USA
}

\begin{abstract}
Measurement of the microgravity acceleration environment on the International Space Station has been accomplished by two accelerometer systems since 2001. The Microgravity Acceleration Measurement System records the quasi-steady microgravity environment, including the influences of aerodynamic drag, vehicle rotation, and venting effects.

Measurement of the vibratory/transient regime, comprised of vehicle, crew, and equipment disturbances, has been accomplished by the Space Acceleration Measurement System-II. Until the arrival of the Columbus Orbital Facility and the Japanese Experiment Module, the location of these sensors, and therefore, the measurement of the microgravity acceleration environment, has been limited to within the United States Laboratory.
\end{abstract}

Japanese Aerospace Exploration Agency has developed a vibratory acceleration measurement system called the Microgravity Measurement Apparatus which will be deployed within the Japanese Experiment Module to make distributed measurements of the Japanese Experiment Module's vibratory acceleration environment. Two Space Acceleration Measurement System sensors from the United States Laboratory will be re-deployed to support vibratory acceleration data measurement within the Columbus Orbital Facility. The additional measurement opportunities resulting from the arrival of these new laboratories allows Principal Investigators with facilities located in these International Space Station research laboratories to obtain microgravity acceleration data in support of their sensitive experiments. The Principal Investigator Microgravity Services project, at NASA Glenn Research Center, in Cleveland, Ohio, has supported acceleration measurement systems and the microgravity scientific community through the processing, characterization, distribution, and archival of the microgravity acceleration data obtained from the International Space Station acceleration measurement systems. This paper summarizes the PIMS capabilities available to the International Space Station scientific community, introduces plans for extending microgravity analysis results to the newly arrived scientific laboratories, and provides summary information for known microgravity environment disturbers.

\section{INTRODUCTION}

The NASA Glenn Research Center (GRC) Principal Investigator Microgravity Services (PIMS) project supports various acceleration measurement systems and, therefore, the microgravity scientific community by providing an infrastructure for the processing, characterization, distribution, and archival of the microgravity acceleration data obtained on-board the International Space Station (ISS). The microgravity acceleration data from the ISS includes measurements from the Japanese Aerospace Exploration Agency (JAXA) and the National Aeronautics and Space Administration (NASA).

Since its advent in the early 1990's in support of microgravity dedicated Space Shuttle missions, the PIMS project has continued its support of microgravity researchers during ISS operations by identifying microgravity acceleration disturbance sources related to vehicle systems, experiment systems, and crew activity. The identification of microgravity acceleration disturbance sources is useful to Principal Investigators (PIs) whose experiments were exposed to the disturbances as well as to future PIs who need to understand the microgravity environment under which their experiment will ultimately operate.[1] Coincident with the build up and growth of the ISS, the acceleration data obtained and archived by PIMS have found increased utility within the ISS vehicle 
community as a tool to assist in the analysis of significant microgravity events and their effect on the overall ISS structure. The design of data analysis techniques, data processing techniques, and the creation of displays per user requirements further enhance an investigator's ability to understand the results of their experiment [2] and further enhance the ability to perform ISS structural analysis. The arrival of the European Space Agency's Columbus laboratory (February, 2008 STS-122) and JAXA's Kibo pressurized module (May, 2008 STS-124) increases microgravity experimentation opportunities on the ISS and brings with it a corresponding need for expanded acceleration measurement capabilities and expanded acceleration data analysis requirements for both the scientific community and the ISS vehicle community.

\section{MICROGRAVITY ENVIRONMENT}

The microgravity acceleration environment of an orbiting spacecraft in low earth orbit is a complex phenomenon. Experiment operation, life-support systems, equipment operation, crew activities, aerodynamic drag, gravity gradient, and rotational effects all contribute to form the overall microgravity environment. Measurement and analysis of the microgravity acceleration environment can be considered as made up of three components: quasisteady, vibratory, and transient components (see Figure 1). The instruments available to measure each of these components each have their own unique set of requirements.

The quasi-steady component responses are composed of those accelerations whose frequency is less than the lowest natural structural frequency of the vehicle. Those accelerations vary over long periods of time, typically longer than a minute. The PIMS definition of the quasi-steady regime for data analysis is disturbance content below $0.01 \mathrm{~Hz}$. The quasi-steady component, because of its low frequency nature, can be measured in a single location on the ISS. Using rigid body assumptions, the location of the vehicle's overall center of gravity, the measurement location, and the location of interest, the quasi-steady component can be mathematically predicted at any location within the vehicle.

The vibratory component response is composed of those accelerations, which are oscillatory in nature and whose frequencies are greater than or equal to the lowest natural structural frequency of the vehicle. They are harmonic and periodic in nature with a characteristic frequency. The PIMS definition of the vibratory regime frequency range for data analysis is 0.01 to $300 \mathrm{~Hz}$. The transient component is composed of those accelerations that last for a short period of time, and are non-periodic. The frequency content varies over the entire frequency spectrum of the data [3]. Unlike the quasi-steady component, the vibratory and transient components need to be measured near the location of interest; no reliable mathematical predictions can be made relative the propagation of a vibratory/transient disturbance through the vehicle.

\section{BASIC OPERATIONAL PHILOSOPHY}

The PIMS operational philosophy addresses several challenges for each measurement system. Acquisition of Signal (AOS) and Loss of Signal (LOS) data streams are automatically merged, providing a time contiguous data set for the sensors. The PIMS acceleration data servers make this contiguous data available via the Internet to all users. Further, the long operational period for the acceleration measurement systems (SAMS and MAMS in particular) requires the ability to accommodate a large volume of data for each measurement system and make that large volume of data readily available. Finally, each SAMS-II accelerometer will not always be actively acquiring and transmitting data. Power profiles and operational issues can result in a limited set of acceleration data to be measured and subsequently available for a given period of time. As a result, a varying active accelerometer configuration profile is created. The PIMS acceleration data archives provide a profile for each operational month to indicate which systems and sensors were operational at a particular time (see Figure 2). In the case of vibratory/transient sensors, the cutoff frequencies are specified as well. This provides the users of the acceleration data a quick assessment of the availability of data and the data's characteristics.

The acceleration data from each sensor system are expected to continue to support the scientific community and the vehicle community throughout the remainder of the ISS program. As a result, the underlying operational philosophy needs to continue to address basic, core functions while allowing flexibility to address needs that develop over the course of operations. With this issue in mind, a core set of functions and capabilities are in place to support analysis requirements. These core capabilities are based on operational experience acquired by PIMS during microgravity-dedicated STS missions and during real-time and offline operations with the ISS. The flexibility in the PIMS analysis systems allows for straightforward development and implementation of additional analysis capabilities as required. The origin of such additional requirements will be PI and vehicle specific needs not addressed by the core functions and 
operational enhancements identified by PIMS during the course of operations.

All the acceleration data processed and archived by PIMS are stored in a time and sensor based directory hierarchy as shown in Figure 3. The time based storage allows users to easily access data based on a particular time period of interest. Each available sensor is displayed at the lowest level of the time hierarchy so that data from multiple sensors can be easily obtained.

All PIMS acceleration data are stored in the Space Station Analysis coordinate system or the ISS United States Orbital Segment Analysis coordinate system. The Space Station Analysis frame has its origin at the geometric center of the Integrated Truss Segment. The positive $\mathrm{X}$-axis is in the direction of flight, the positive $\mathrm{Y}$-axis is in the starboard direction, and the positive Zaxis is pointed toward earth. The selection of a common coordinate system allows for much easier comparison of data from different locations in the various laboratories. [4]

\section{ACCELEROMETER DESCRIPTION}

The ability to measure the three components of the overall microgravity environment is handled by two different types of measurement systems: quasi-steady and vibratory. On the ISS, quasi-steady acceleration measurements are obtained by the Microgravity Acceleration Measurement System's (MAMS) Orbital Acceleration Research Experiment (OARE) Sensor Subsystem (OSS). The nature of the quasi-steady environment allows this single measurement to handle the quasi-steady measurement requirements of the entire ISS.

Conversely, the vibratory and transient components of the overall acceleration environment are measured by three unique systems located throughout the ISS laboratory environment. The MAMS High-Resolution Accelerometer Package (HiRAP) provides one of the vibratory/transient measurement tools. HiRAP is capable of measuring vibratory disturbances up to 100 Hz. The Space Acceleration Measurement System (SAMS) represents the second vibratory/transient measurement system on the ISS. Unlike HiRAP which provides a single sensor in a single location within the United States Laboratory Module (USLab), SAMS provides a distributed measurement system capable of making acceleration measurements within the Columbus Facility, the Kibo pressurized module, and the USLab. Each SAMS sensor has an adjustable filter setting that allows filtering of the measured data from a minimum cutoff frequency of $25 \mathrm{~Hz}$ to a maximum cutoff frequency of $400 \mathrm{~Hz}$. The third vibratory/transient measurement system is JAXA's Microgravity Measurement Apparatus (MMA) which began operations on the ISS in August, 2008. The MMA has three remotely located sensors available for obtaining vibratory/transient acceleration measurements within the Kibo module. Like SAMS, each MMA has an adjustable filter setting. For MMA, the minimum cutoff frequency is $10 \mathrm{~Hz}$ and the maximum cutoff frequency is $300 \mathrm{~Hz}$.

DATA MEASUREMENT ONBOARD THE ISS Configuration - Prior to Columbus Facility Arrival Since SAMS and MAMS arrived on the ISS during flight 6A in April, 2001, four serialized SAMS Sensor Enclosure (SE) heads have been utilized for the majority of SAMS-based acceleration measurement: 121F02 through 121F05. Each sensor head has a defined coordinate system whose location and orientation is defined with respect to the Space Station Analysis Coordinate System [4]. Each origin is defined at the triaxial center point of the three accelerometers that comprise the head. SAMS SE 121F02 was mounted in the SAMS International Subrack Interface Standard (ISIS) drawer 1 in the Expedite the Processing of Experiments to the Space Station (EXPRESS) Rack 1. Each SAMS ISIS drawer contains a single Electronics Enclosure (EE) that provides the connectivity for one or two SAMS Sensor Enclosures (SE). The SE is the SAMS hardware that contains the actual accelerometer electronics. EXPRESS Rack 1 was located in the overhead bay 2 of the US Laboratory Module (LAB1O2). Head 121F03 was mounted on the lower Z panel assembly below EXPRESS Rack 2. EXPRESS Rack 2 was located in overhead bay 1 of the US laboratory Module (LAB1O1). Head 121F04 was mounted on the lower $\mathrm{Z}$ panel assembly below EXPRESS Rack 1; head 121F05 was mounted on the bracket around the upper $\mathrm{Z}$ panel light assembly of EXPRESS Rack 2. Figure 4 shows the location of each SAMS SE for EXPRESS Racks 1 and 2. The control unit for the SAMS sensors is located in EXPRESS Rack 4, port location bay 2.

A fifth SAMS sensor head is owned by the Microgravity Science Glovebox (MSG) and is available on an as-requested basis from MSG to provide localized acceleration measurement of the MSG work volume. With the completion of flight ULF-2, the MSG SAMS head represents new SAMS technology and is called a TSH-ES (Triaxial Sensor Head-Ethernet Standalone); the TSH-ES does not require a SAMS EE to communicate with the SAMS ICU. Consequently, less MSG work volume is required for acceleration measurement. The sensor itself is installed within the MSG work volume and its location and orientation are 
a function of the glovebox investigation requesting acceleration data support. MSG was located in the starboard bay 3 location.

The MAMS-OSS and MAMS HiRAP instruments were located in the middeck lockers 3 and 4 of EXPRESS Rack 1. The origin of MAMS-OSS coordinate system is located at the center of gravity of the proof mass. Figure 4 and Figure 5 show the location of MAMSOSS and MAMS HiRAP in EXPRESS Rack 1 relative to the SAMS-SEs and within the US Lab. The SAMS and MAMS sensors have recorded over 2 terabytes of data since their initial activations in April, 2001.

\section{Configuration - Columbus Facility February 2008} The arrival of the Columbus Orbital Facility in February, 2008 eventually resulted in the movement of the MSG rack from its location in the USLab to COL1F2 in the COF. It also resulted in the movement of ER\#3 from its location at Overhead bay 3 to COL1A1 in the COF. These rack re-locations are significant for two reasons. First, the movement of the MSG to the COF automatically provides a SAMS acceleration measurement capability within the COF. The second reason involves ER\#3 which has included in its configuration an embedded SAMS EE. This embedded ER\#3 EE provides an additional SAMS acceleration measurement capability within the COF by simply connecting a SAMS SE to the ER\#3 embedded EE.

Configuration - Kibo Pressurized Module June 2008 The arrival of the MMA system to the ISS in June, 2008 represents another milestone in the acceleration measurement capabilities on the ISS. MMA began its operations in August, 2008 and consists of three sensors, all located within the Kibo pressurized module. The MMA acceleration data will be processed and archived into the PIMS acceleration data servers for analysis and distribution. The MMA sensors are located at MMA racks locations JPM1A2 and JPM1A3. The entire laboratory configuration is provided in Figure 6.

\section{Planned Future Configuration Changes}

The next major planned acceleration measurement configuration change involves the movement of one of the SAMS ISIS drawers from the USLab to the ER\#4 located in the JEM. This movement will result in the ability to use SAMS to simultaneously measure the ISS acceleration environment in each laboratory.

\section{DATA ANALYSIS TECHNIQUES AND PROCESSING}

The PIMS project has developed several techniques to analyze the acceleration environment on the ISS. The particular technique employed is dictated by the information to be gleaned from the data. Time domain techniques are use predominantly for quasi-steady analysis while frequency domain analysis is used for the higher frequency data from SAMS. Prior to performing SAMS analysis, it is standard practice to de-mean vibratory data; focus is consequently placed on the dynamic, oscillatory components of the acceleration environment.

The basis for the frequency domain analyses that are performed is the acceleration power spectral density (PSD). The PSD is a function that quantifies the distribution of power in a signal with respect to frequency. When properly applied, it serves as a powerful tool to help identify and quantify oscillatory components of the acceleration environment. An introductory presentation of the PSD along with other vibratory analysis techniques is given in [5].

Mathematical foundation and important details are given in $[6,7]$.

Displays of acceleration PSDs can give much useful information, but keen insight to the dynamic nature of the acceleration spectrum aboard a manned spacecraft comes when numerous PSDs are arrayed as a function of time or combined in the form of a two-dimensional histogram. A spectrogram is a three-dimensional plot that shows PSD magnitude (represented by color) versus frequency versus time. It is a powerful qualitative tool for characterizing long periods of data. Boundaries and structure in both time and frequency become apparent with this type of display. When acceleration PSDs are laid out versus time for regular 8-hour intervals, the resulting displays are referred to as roadmap spectrograms or simply roadmaps.

Alternatively, rather than show PSDs versus time, they can be combined in the form of a two-dimensional histogram as discussed in [5]. A display of acceleration spectra in this form is referred to here as a Principal Component Spectral Analysis (PCSA) plot. Its name stems from the fact that it serves to summarize magnitude and frequency variations of key spectral contributors for a relatively long period of time. [3]

\section{PIMS WEB SITE CAPABILITIES}

The primary interface to the acceleration data provided by PIMS and the various ISS acceleration measurement systems is the PIMS ISS web site. This public web site (http://pims.grc.nasa.gov) provides links to real-time acceleration data, as well as analyzed acceleration data 
products. A scrolling window on the main PIMS web page provides operational details on the currently active accelerometers. This is primarily an AOS/LOS indication for the individual sensors.

Through the tab Current Real Time, the user gains access to all of the acceleration data plots currently generated by the PIMS ground system for a particular sensor. While all of the time and frequency domain acceleration data plots are listed as an option, the default data plot for all vibratory/transient acceleration measurement systems is typically the color spectrogram. The color spectrogram is utilized for its ability to qualitatively display, in a single plot, a large amount of acceleration data over a relatively long period of time. Based on the requests of investigators, additional plots can be enabled by the PIMS ground system and displayed in a fashion similar to the color spectrograms. For the quasi-steady measurements from the MAMS OSS sensor, a time domain plot of filtered data is available. This plot typically displays the MAMS OSS data at multiple locations (recall quasisteady data can be mathematically predicted at points within the ISS other than the as-measured location), including the as-measured location and the ISS center of gravity. Like the spectrogram's selection in the frequency domain, the MAMS filtered data versus time is selected because the plot displays over three hours of MAMS OSS data in a single view. In the case of quasisteady data, this represents over two orbits of the quasisteady acceleration data signal.

The tab Accel Archive provides access to all of the acceleration data received and process by the PIMS ground systems. The link itself provides detailed instructions on how to download the acceleration data files and test files to verify the user's ability to properly read the acceleration data files once downloaded. Another important feature available through this link is the roadmap plots, generated for both quasi-steady and vibratory/transient acceleration measurement systems. Like the default real time plots, the color spectrogram is used for vibratory/transient acceleration data and the filtered time domain plot is used for the MAMS OSS data. Each plot generated is eight hours in duration and is intended to highlight at a high level disturbances and characteristics of the microgravity acceleration environment of that eight hour period.

The final tab to be discussed is the Handbook tab which represents a summary of characterized microgravity events in a common display format. The link itself is separated into a quasi-steady set of handbook results and a vibratory/transient set of handbook results. The vibratory/transient section is further subdivided into Crew Activity, Experiment Equipment, and Vehicle headings. The concept behind the handbook page is to first qualify and then quantify the disturbance under investigation. The common display format was developed to provide standard information for each analyzed disturbance, including the sensor characteristics, the time frame for which the analysis was performed, and the physical location of the disturbance source. Each disturbance has a quantification step aimed at extracting a g-level with the event under investigation. All of the above information is intended to provide summary data about known microgravity conditions on the ISS. Investigators can utilize this information to make informed decisions about operations planning and postexperiment data analysis.

Based on the handbook web page discussed previously, four representative sets of analysis have been selected for further presentation. The first handbook page selected is for Resistive Device (RED) exercise which is a multi-purpose piece of equipment used as one part of the crew member exercise regimen and is used to perform squat, deadlift, and rowing type exercises. As a consequence, this is a disturbance signature that is present on a daily basis. Figure 7 shows a color spectrogram of SAMS data of forty-five minutes in duration. The vertical traces in the plot indicate transient disturbances associated with each exercise. Figure 8 is a Root Mean Square (RMS) acceleration versus time plot that serves to quantify the accelerations associated with each exercise movement.

The second handbook page is for the initial Automated Transfer Vehicle (ATV) docking that occurred in April 3 , 2008. Figure 9 is an acceleration vector magnitude versus time plot of SAMS data that serves to quantify the ATV's initial contact with the ISS. The initial contact can be seen at approximately the six minute mark of the plot and presents a $12.9 \mathrm{mg}$ disturbance to the overall ISS structure. Obviously, vehicle dockings are another disturbance source that cannot be avoided operationally and their characteristic signatures need to be well understood. Similar types of analysis have been performed for Russian Soyuz and Progress vehicles as well as Space Shuttle dockings to the ISS.

A third unavoidable microgravity disturber is reboosts of the ISS. The analysis of the reboosts, whether from the Progress vehicle or the ATV, provides two important pieces of information to two distinct parts of the ISS program. The first piece of information is the more familiar microgravity disturbance level associated with the event. The time domain plot of filtered MAMS data in Figure AAA shows the acceleration

\section{American Institute for Aeronautics and Astronautics}


disturbance imparted on the ISS in the $\mathrm{x}$-axis direction. The second piece of information is a quantification of the purpose of the reboost itself, an increase in the velocity of the ISS. The ISS is continually decelerating, causing the orbit to slowly decay. Reboosts impart a delta- $\mathrm{V}$ on the structure to restore the orbit to its desire state. The ATV reboost in Figure 10 lasted approximately twenty-one minutes and provided an increase in the ISS velocity of just over 4 meters/second.

The final microgravity handbook page for discussion is provided in Figure 11 and Figure 12. The color spectrogram of MAMS HiRAP data in Figure 11 shows two vibratory signatures starting around the 17 minute mark and stopping around the 23 minute mark. These represent the components of the Urine Processing Assembly (UPA) installed during flight ULF-2 as part of the Water Recovery System (WRS). The handbook page is significant because it illustrates a disturbance signature generated by crew life support systems.

Figure 12 illustrates two PSD plots used to demonstrate the individual components of the Distillation Assembly and their contribution to the overall environment.

\section{SUMMARY}

The recent arrival of the Columbus Orbital Facility and the Kibo Pressurized Module presents new

opportunities on the ISS for microgravity research and microgravity acceleration measurement. The arrival of JAXA's MMA, coupled with the existing measurement capabilities of SAMS and MAMS allow for distributed acceleration measurement within each of the ISS laboratories in support of microgravity PI's. The Principal Investigator Microgravity Services project, at NASA Glenn Research Center, in Cleveland, Ohio, will continue to support acceleration measurement systems and the microgravity scientific community through the processing, characterization, distribution, and archival of the microgravity acceleration data obtained from the International Space Station.

\section{ACKNOWLEDGMENTS}

The authors would like to acknowledge a number of people whose work contributed significantly to this paper. Significant contributions were made in the area of software development, the Microgravity Analysis Software System (MASS), which enabled both MAMS and SAMS to acquire acceleration data aboard the ISS, process, analyze, and display the data on the PIMS website. This same set of capabilities has been extended recently to include JAXA's MMA system as well.

\section{REFERENCES}

1. Rogers, M.J.B., Hrovat, K., McPherson K., Moskowitz M., Reckart, T., Accelerometer Data Analysis and Presentation Techniques, NASA Technical Memorandum TM-113173, Sept 1997

2. McPherson, K., Hrovat, K., "Measurement and Data Distribution for Microgravity Accelerations on the International Space Station”, IAF-99-J.2.05, 50th International Astronautical Congress, Oct 1999, Amsterdam, Netherlands.

3. Jules, K., et al, “A Status Report on the Characterization of the Microgravity Environment of the International Space Station”, IAC-02-J.6.01, $52^{\text {nd }}$ International Astronautical Congress, October, 2003, Bremen, Germany

4. Jules, K., et al, "International Space Station Increment4/5 Microgravity Environment Summary Report”, NASA/TM-2003-212460, July 2003

5. Hrovat, K., $5^{\text {th }}$ Annual Microgravity Environment Interpretation Tutorial (MEIT), NASA-PIMS, Volume 1, Section 7, March 5-7, 2002.

6. Alan V. Oppenheim, Ronald W. Schafer, Digital Signal Processing, Prentice-Hall, Inc., NJ, 1995.

7. Julius S. Bendat, Allan G. Piersol, Random Data: Analysis and Measurement Procedures, WileyInterscience, New York, 1986.

\begin{tabular}{|c|c|}
\hline KEY ACRONYMS & DEFINITION \\
\hline$\overline{\mathrm{ATV}}$ & Automated Transfer Vehicle \\
\hline $\mathrm{COF}$ & Columbus Orbital Facility \\
\hline ER & EXPRESS rack \\
\hline GRC & Glenn Research Center \\
\hline HiRAP & High Resolution \\
\hline & Accelerometer Package \\
\hline ISIS & $\begin{array}{l}\text { International Subrack Interface } \\
\text { Standard }\end{array}$ \\
\hline ISS & International Space Station \\
\hline JAXA & $\begin{array}{l}\text { Japanese Aerospace } \\
\text { Exploration Agency }\end{array}$ \\
\hline MAMS & $\begin{array}{l}\text { Microgravity Acceleration } \\
\text { Measurement System }\end{array}$ \\
\hline MMA & $\begin{array}{l}\text { Microgravity Measurement } \\
\text { Apparatus }\end{array}$ \\
\hline MSG & $\begin{array}{l}\text { Microgravity Science } \\
\text { Glovebox }\end{array}$ \\
\hline OARE & $\begin{array}{l}\text { Orbital Acceleration Research } \\
\text { Experiment }\end{array}$ \\
\hline OSS & OARE Sensor Subsystem \\
\hline PAD & PIMS Acceleration Data \\
\hline PCSA & $\begin{array}{l}\text { Principal Component Spectral } \\
\text { Analysis }\end{array}$ \\
\hline PIMS & $\begin{array}{l}\text { Principal Investigator } \\
\text { Microgravity Services }\end{array}$ \\
\hline PSD & Power Spectral Density \\
\hline RED & Resistive Device \\
\hline RMS & Root Mean Square \\
\hline SAMS & $\begin{array}{l}\text { Space Acceleration } \\
\text { Measurement System }\end{array}$ \\
\hline
\end{tabular}


SE

STS

TMF

UPA

USLab

WRS
Sensor Enclosure

Space Transportation System

Trimmed Mean Filter

Urine Processing Assembly

U.S. Laboratory Module

Water Recovery System 
Microgravity is:

- a general label for scientific investigations that exploit near-weightlessness

- one-millionth of Earth's normal gravity

- a reduced gravity environment

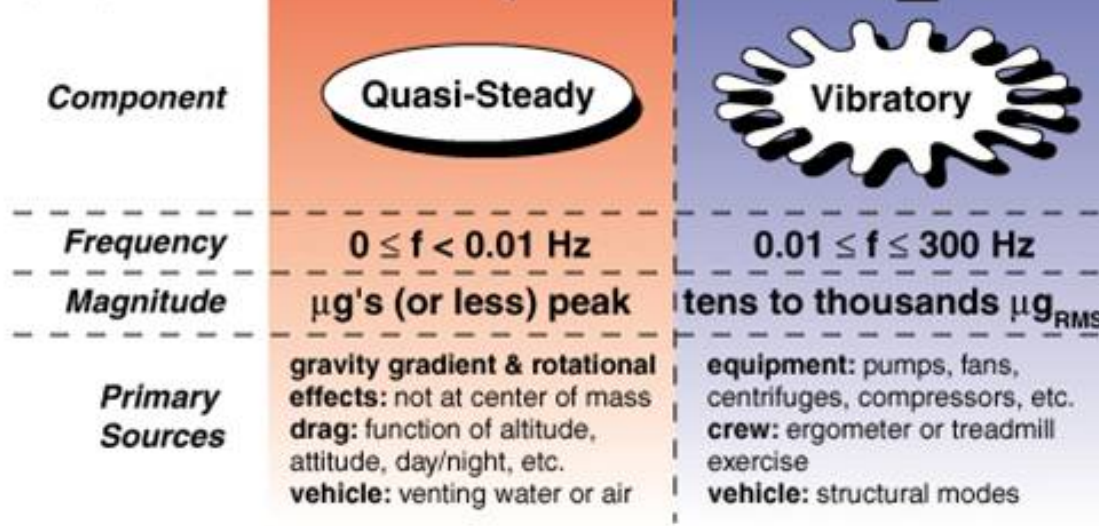

\section{Microgravity Environment}
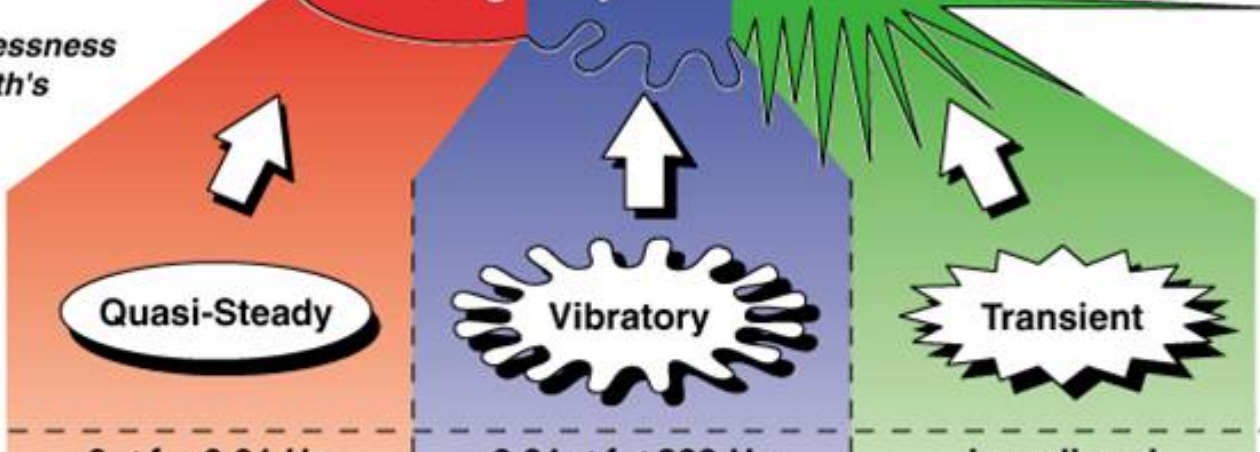

Figure 1 - Components of the Microgravity Environment

PAD Profile for December of 2001 (GMT Days of Year 335 to 365)

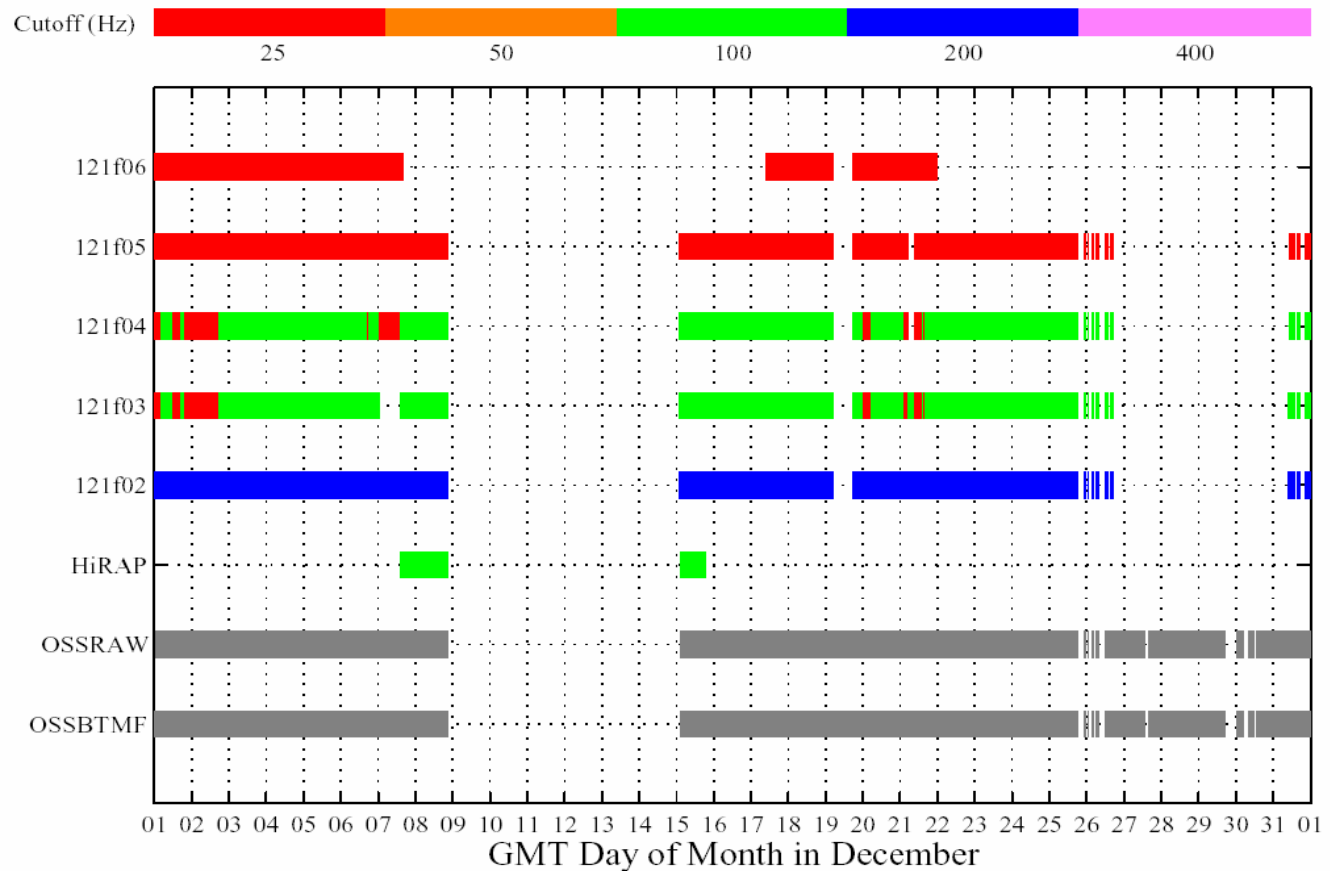

Figure 2 - PIMS Acceleration Data Profile 


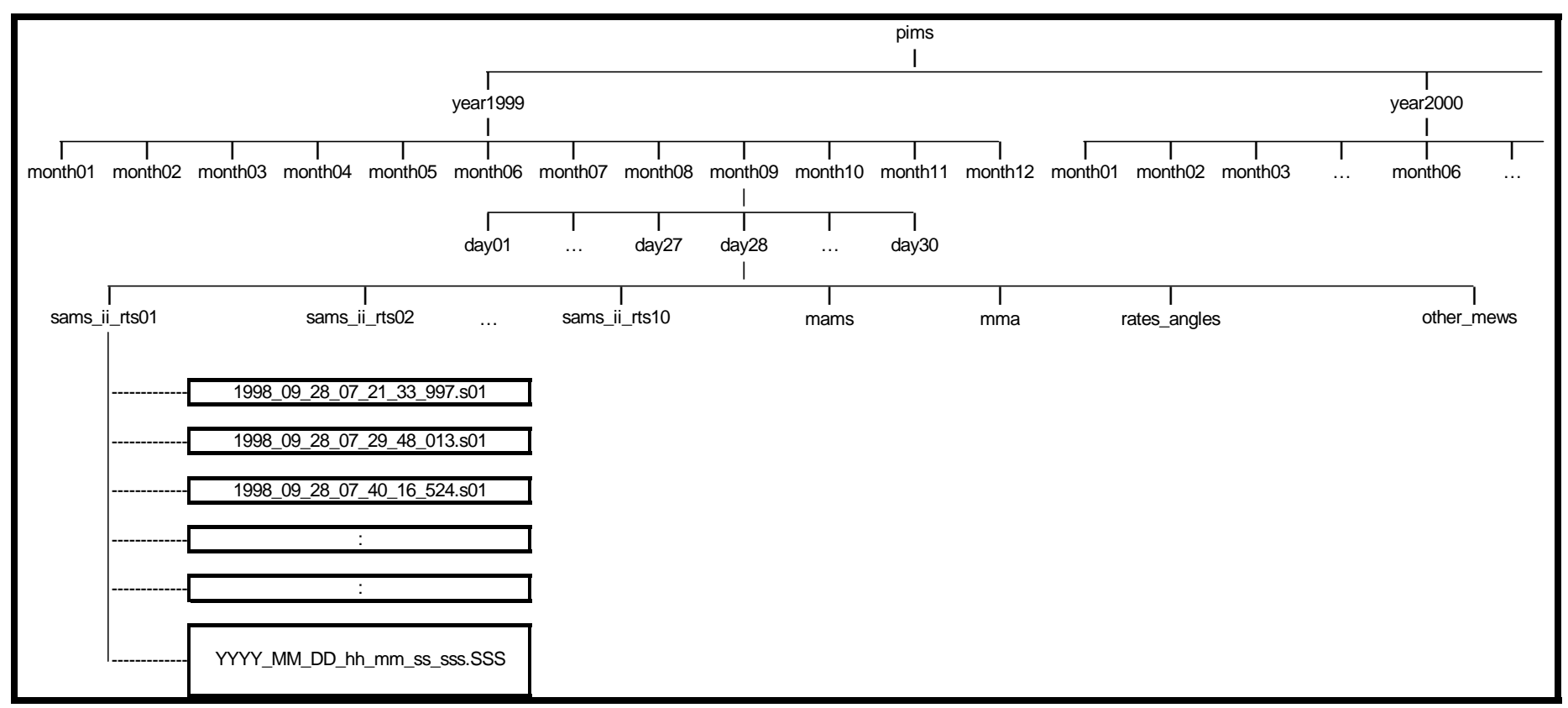

Figure 3 - PIMS Directory Hierarchy

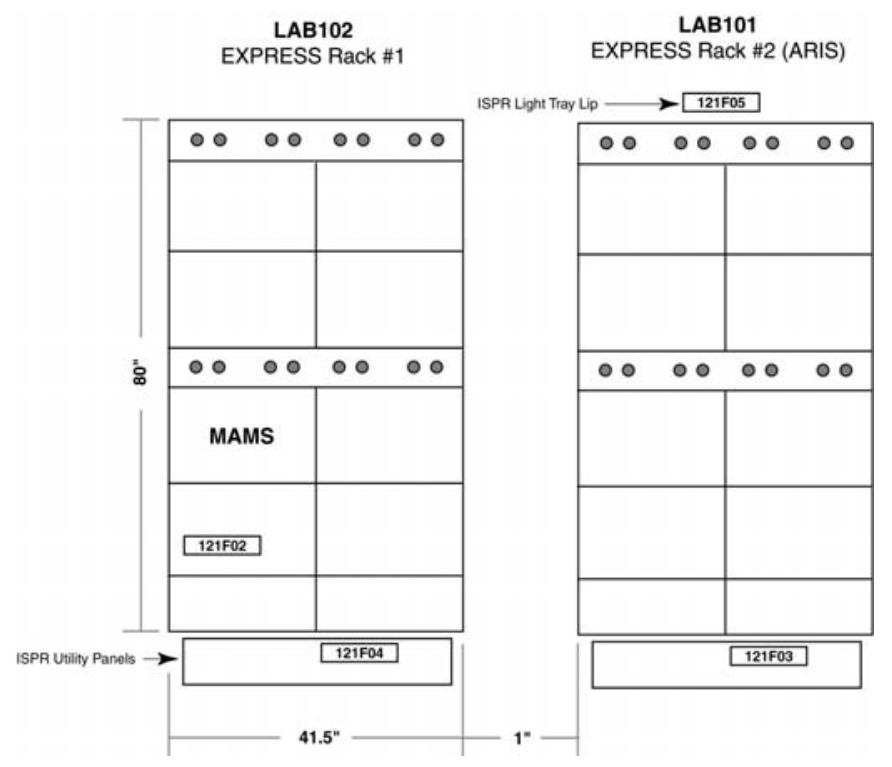

Figure 4 - MAMS and SAMS Accelerometers Locations On/in ER 1 and 2 For Increments 5 And 6 


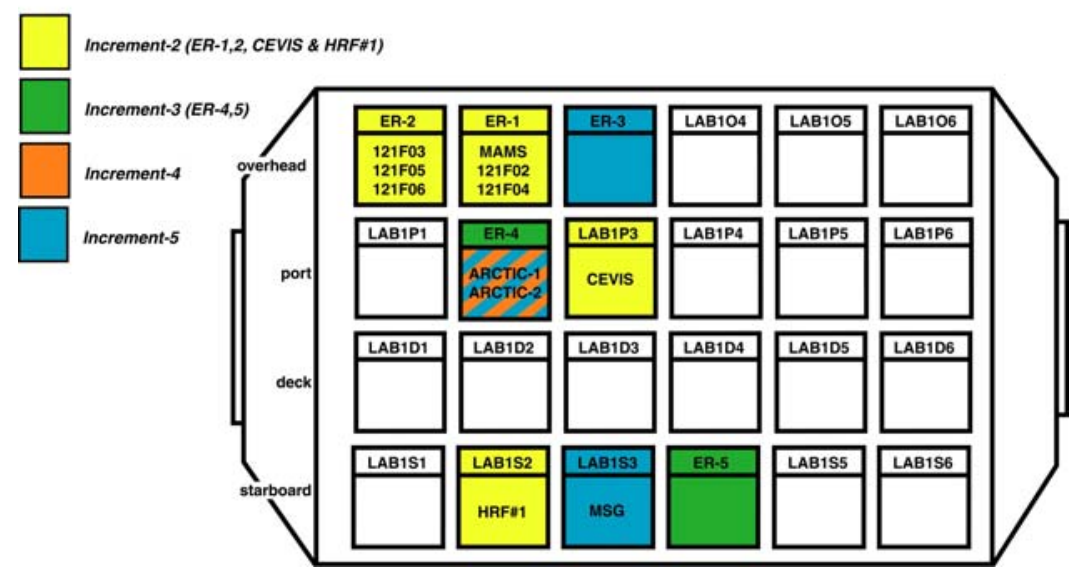

Figure 5 - US LAB Layout Up To Increment-6

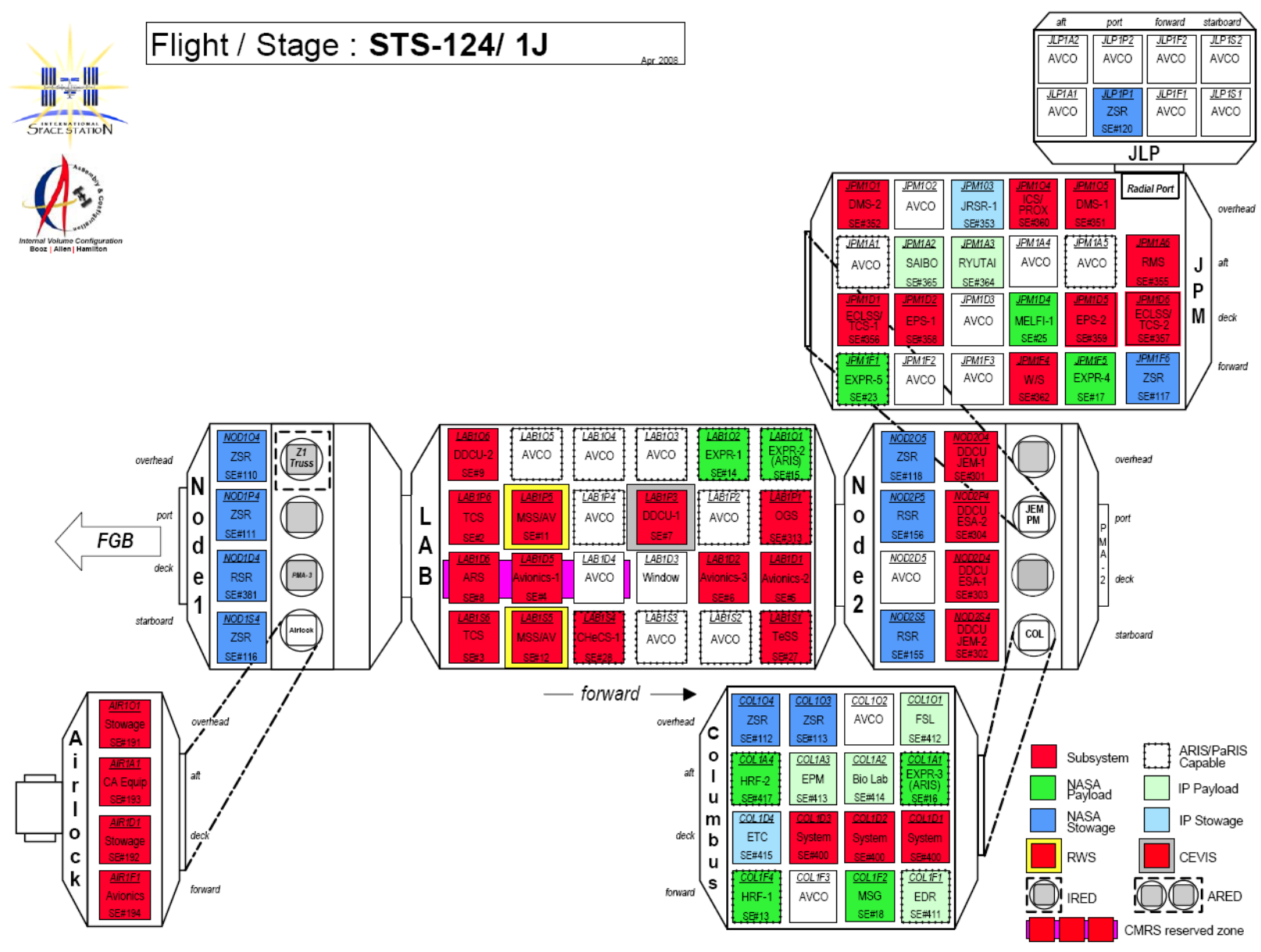

Figure 6 - 1J ISS Laboratory Rack Topology 


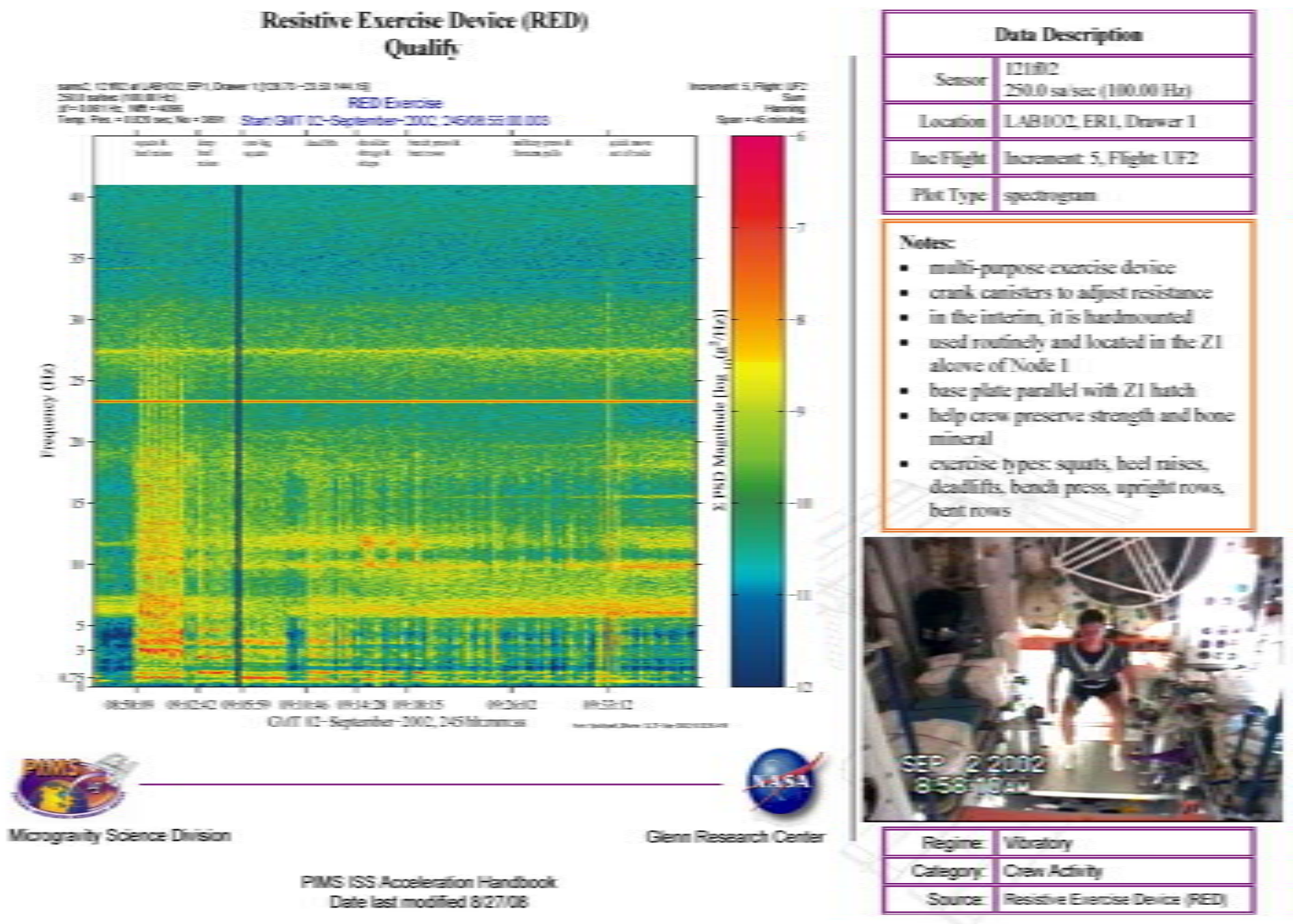

Figure 7 - RED Exercise Spectrogram 


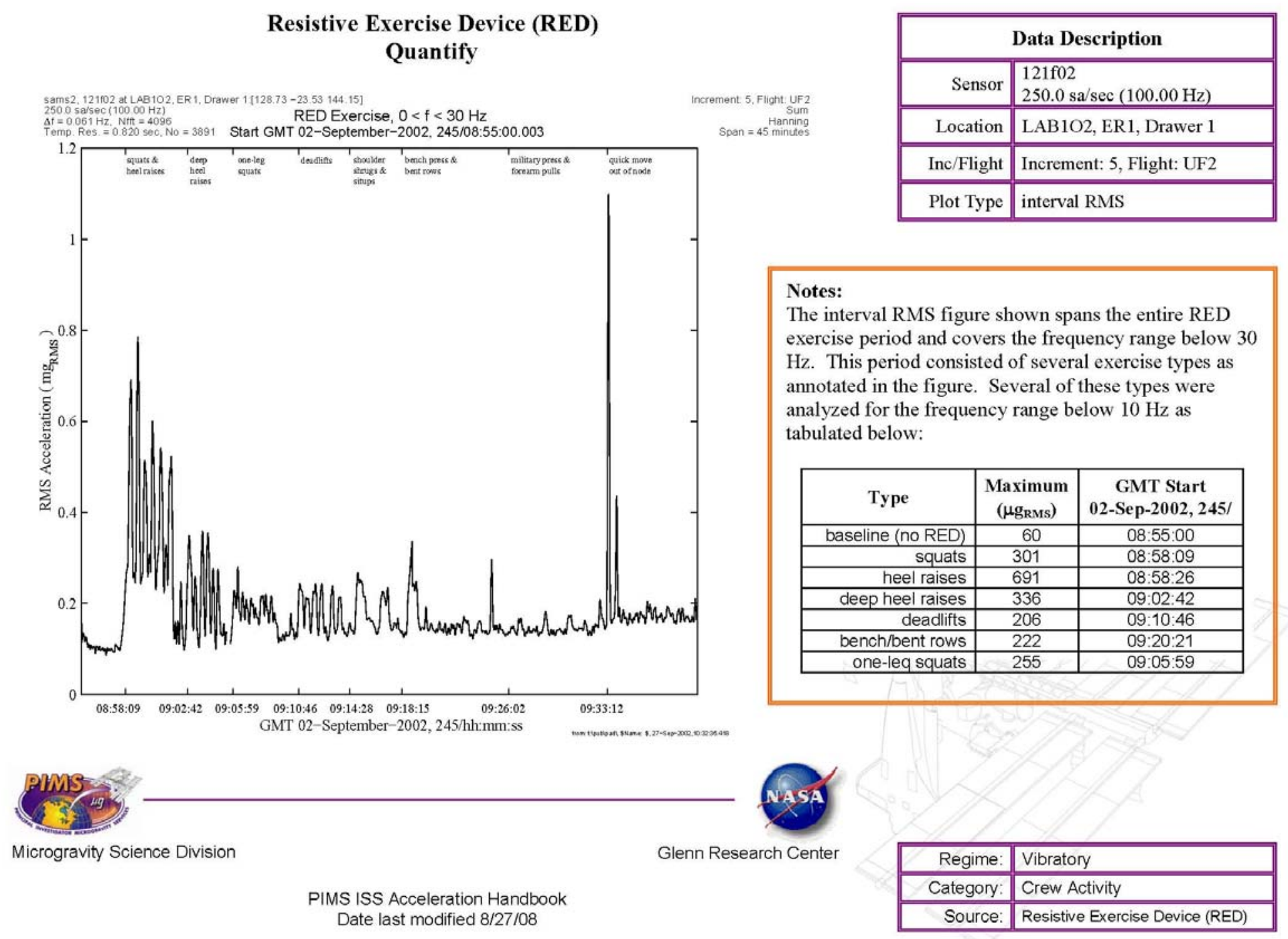

Figure 8 - RED Exercise RMS Acceleration versus Time

12

American Institute for Aeronautics and Astronautics 


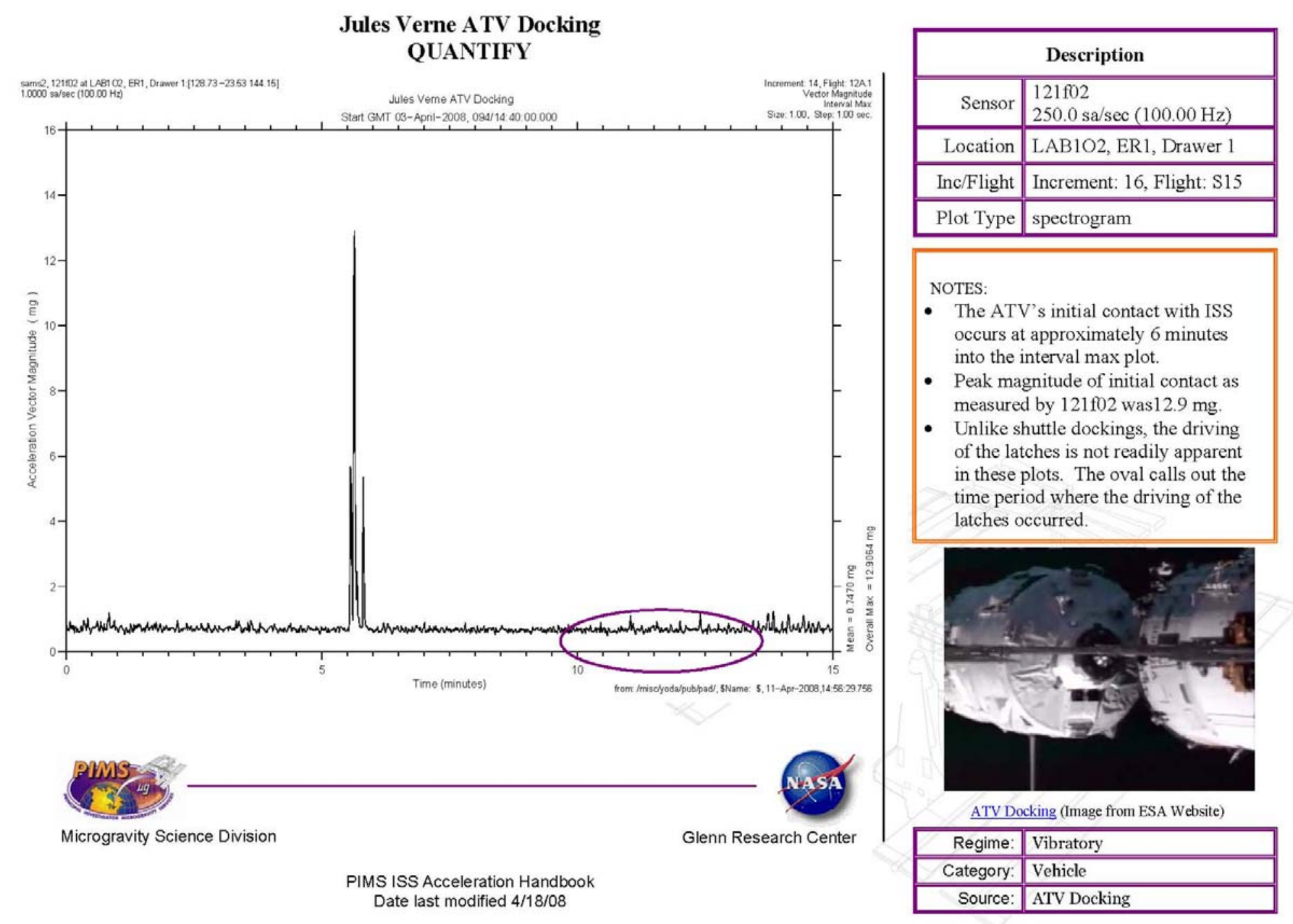

Figure 9 - ATV Docking Acceleration Vector Magnitude

13

American Institute for Aeronautics and Astronautics 


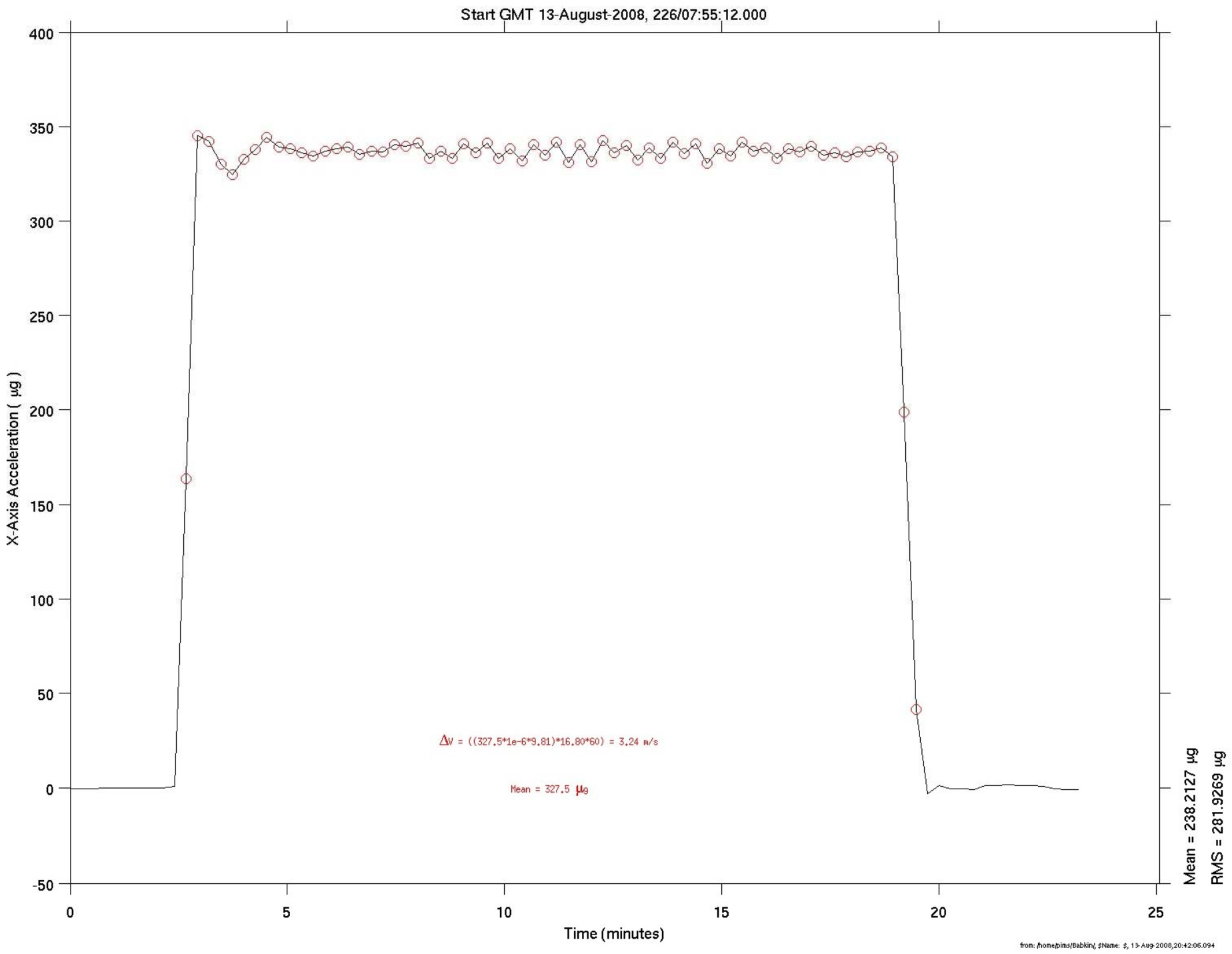

Figure 10 - ATV Reboost GMT 226 
Urine Processing Assembly (UPA)

Qualify

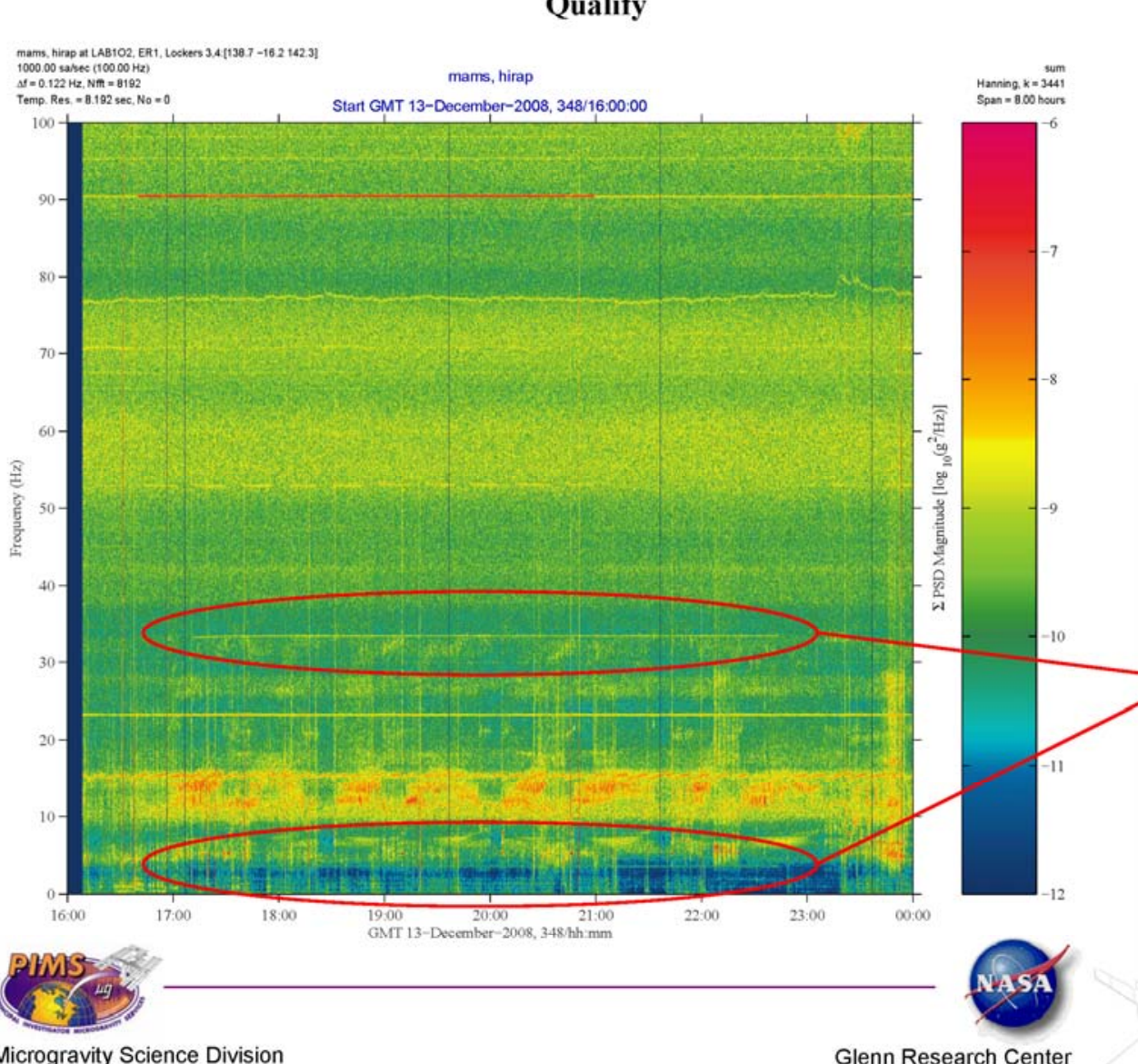

Microgravity Science Division

\begin{tabular}{|r||l|}
\hline \multicolumn{2}{|c|}{ Description } \\
\hline \hline Sensor & $\begin{array}{l}\text { HiRAP } \\
1000.0 \text { sa/sec }(100.00 \mathrm{~Hz})\end{array}$ \\
\hline \hline Location & LAB1O2, ER1, Lockers 3,4 \\
\hline Orientation & $\begin{array}{l}\text { Space Station Analysis } \\
\text { (SSA) }\end{array}$ \\
\hline \hline Inc/Flight & Increment: 18, Flight: ULF2 \\
\hline Plot Type & Spectrogram \\
\hline
\end{tabular}

NOTES:

- The Urine Processing Assembly (UPA) was installed in LABIP4 during ULF-2 as part of the Water Recovery System (WRS).

- The UPA contains 3 known potential microgravity disturbers: Distillation Assembly (DA) centrifuge, Fluids Control \& Pump Assembly (FCPA) and the Pressure Control \& Pump Assembly (PCPA).

- MAMS HiRAP captured a DA centrifuge signature during a 5 hour run on GMT 348 . The spectrogram on the left shows two distinct traces at $3.6 \mathrm{~Hz}$ and $33.3 \mathrm{~Hz}$ during this run. Data acquired from ISS telemetry confirms the DA centrifuge speed near 220 rpm.

- No signature for PCPA is seen, which was known to be operating intermittently at 2500 rpm during the run. $(\sim 2 \mathrm{~Hz})$.

- Whether the FCPA was operating at this time was not determined.

\begin{tabular}{|r|l|}
\hline Regime: & Vibratory \\
\hline \hline Category: & Vehicle \\
\hline \hline Source: & Urine Processing Assembly \\
\hline
\end{tabular}

PIMS ISS Acceleration Handbook Date last modified 12/22/08

Figure 11 - Urine Processing Assembly (UPA) Spectrogram 
Urine Processing Assembly (UPA)

QUANTIFY

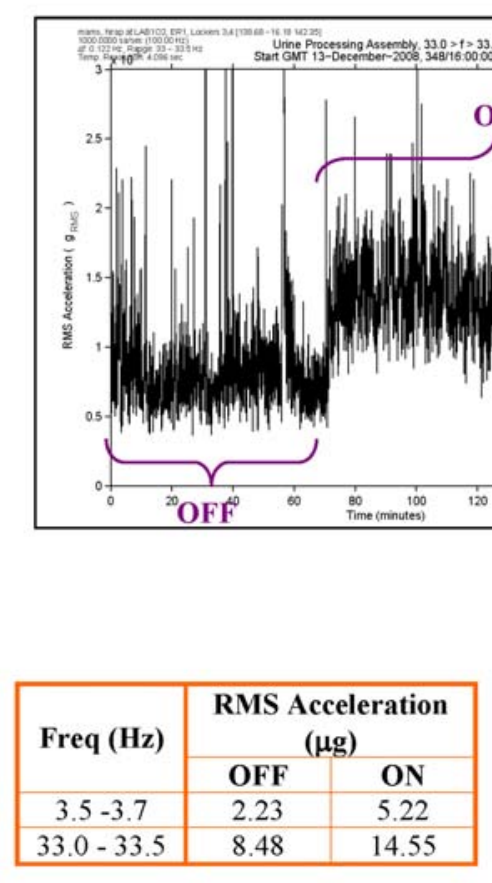

